International Journal of Control

Vol. 00, No. 00, Month 200x, 1-18

Preprint of International Journal of Control, 2014

Vol. 87, No. 11, pp.2372-2382

http://dx.doi.org/10.1080/00207179.2014.921935

\title{
Consensus Control of A Class of Lipschitz Nonlinear Systems
}

\author{
Zhengtao Ding* \\ Control Systems Centre, School of Electrical and Electronic Engineering \\ University of Manchester, Sackville Street Building, Manchester M13 9PL, UK
}

(submitted to Int J Control)

\begin{abstract}
This paper deals with consensus control of a class of nonlinear systems with Lipschitz nonlinearities. Certain features of the Laplacian matrix are explored in the real Jordan form to identify conditions for global consensus control. Under the identified conditions, consensus control and stability of the proposed control are analysed in time-domain through Lyapunov functions in real domain. The proposed control uses relative state information of the system. A simulation study is included to demonstrate the proposed control designs with some simulation results shown.
\end{abstract}

Keywords: consensus control, Lipschitz nonlinearity, Lyapunov function, Laplacian matrix,

\section{Introduction}

With the recent advances in measurements and communication, dynamic systems are often connected together to achieve specified control tasks. In many applications, the subsystems or agents have identical or very similar dynamics, and they are required to achieve the same or similar tasks. In some applications, the information regarding the relative states are measured and control objectives may also be based on the relative states, such as formation control etc. Consensus control is normally referred to the type of control design that achieves the identical control objectives for the subsystems that are connected by a network, based on the information obtained from the network.

The connections between the subsystems are important to the design and analysis of consensus control systems. One significant advance in consensus control is to use tools in graph theory to characterise the network connection, and in particular, the use of Laplacian matrices (Fax and Murray 2004, R.Olfati-Saber and Murray 2004). Consensus control has attracted significant attention in recent years, and many theoretical and practical issues have been analysed and reported in literature (Hong et al. 2006, Ren 2007, Xiang et al. 2009, Li et al. 2010, Wang et al. 2010, Liu et al. 2011, Liu 2011, Su and Huang 2012, Ding 2012, 2013). Many of the results are based on linear system dynamics. The results on consensus control of nonlinear systems often are restricted to local stability, or restricted to certain connections of the network such as the existence of a tree structure in the connection, or certain types of nonlinearities. The difficulty of solving consensus control problem of nonlinear systems is mainly due to certain restrictions in using the information for individual systems. For example, if a subsystem has all the information of its state, and the local control is allowed, the existing nonlinear control strategies may be applied to linearise the dynamics of each of the subsystems. In such a case, consensus control for linear systems can be applied to linearised subsystems. Consensus control of second-order systems with Lipschitz nonlinearities was investigated by Yu et al. (2010), Song et al. (2010). The results shown in ( $\mathrm{Li}$ et al. 2012, 2013), brought to the author's attention

\footnotetext{
*Email: zhengtao.ding@manchester.ac.uk
} 
after submitting the original version of this paper, deal with high-order dynamic systems with Lipschitz nonlinearities with certain types of connections. In particular, Li et al. (2012) obtains the result when the left eigenvector corresponding to the eigenvalue at zero of the Laplacian matrix has no zero elements, and the result shown in ( $\mathrm{Li}$ et al. 2013) are for the connections with undirected graphs that implies that the left eigenvector for the eigenvalue at zero are with positive elements of an identical value.

In this paper, we will systematically investigate consensus control with global stability for a network-connected system with nonlinearity in the system dynamics under a fairly general assumption about the connection structure. The nonlinearities are restricted to being Lipschitz, which allows less restriction on the structure of nonlinearity. For the control design, only the relative information obtained via the network connection is used, without local feedback control of the subsystems. The Laplacian matrix is transformed to the real Jordan form for the stability analysis. By using the real Jordan form, a framework for stability analysis of consensus control systems is developed for using Lyapunov functions in real domain, and the framework is then used to explore the conditions on the nonlinearity for the global consensus control. When there is a spanning tree in the network connection, conditions are identified for the Lipschitz nonlinearity in the system. The conditions depend on the eigenstructure of the Laplacian matrix. A more stringent condition is required when there are multiple eigenvalues in the Laplacian matrix than the case of the Laplacian with only distinct eigenvalues. The significance of this research is to provide a measure on nonlinearity that can be tolerated under consensus control strategy with the global stability. A simulation study has been carried out with the results shown in the paper.

\section{Problem Statement}

In this paper, we consider a set of $N$ nonlinear subsystems, of which the subsystems are with identical dynamics described by

$$
\dot{x}_{i}=A x_{i}+\phi\left(x_{i}\right)+B u_{i}
$$

for $i=1, \ldots N$, where $x_{i} \in \mathbb{R}^{n}$ is the state vector of the subsystem, $u_{i} \in \mathbb{R}^{m}$ is the input of the $i$ th subsystem, $A \in \mathbb{R}^{n \times n}$ and $B \in \mathbb{R}^{n \times m}$ are constant matrices with $(A, B)$ controllable, and $\phi: \mathbb{R}^{n} \rightarrow \mathbb{R}^{n}$ is a Lipschitz nonlinear function with the Lipschitz constant $\gamma$, i.e., for any two constant vectors $a, b \in \mathbb{R}^{n}$, we have

$$
\|\phi(a)-\phi(b)\| \leq \gamma\|a-b\|
$$

The connections between the subsystems are specified by a directed graph $\mathcal{G}$ which consists of a set of vertices denoted by $\mathcal{V}$ and a set of edges denoted by $\mathcal{E}$. A vertex represents a subsystem, and each edge represents a connection. Associated with the graph, its adjacency matrix $Q$ with elements $q_{i j}$ denotes the connections such that $q_{i j}=1$ if there is a connection from subsystem $j$ to subsystem $i$, and $q_{i j}=0$ otherwise. The Laplacian matrix $L=\left\{l_{i j}\right\}$ is commonly defined by

$$
\begin{aligned}
& l_{i j}=-q_{i j}, \text { if } j \neq i, \\
& l_{i i}=\sum_{j=1, j \neq i}^{N} q_{i j} .
\end{aligned}
$$

From the definition of the Laplacian matrix, it is clear that

$$
L 1=0
$$


where

$$
\mathbf{1}=[1, \ldots, 1]^{T} \in \mathbb{R}^{N}
$$

This implies that the Laplacian matrix has 0 as an eigenvalue, associated with the eigenvector 1.

Assumption 1: The eigenvalue of the Laplacian matrix at 0 is a single eigenvalue.

Remark 1: This assumption is essential for consensus control. In terms of the network collections, this condition implies that the network has a spanning tree to connect any two subsystems in the system.

The consensus control problem considered in this paper is to design a control strategy, using the relative state information to ensure that all the subsystems converge to an identical trajectory.

\section{Consensus Control}

In this section, we propose a control design using the relative state information. The control input takes the structure

$$
u_{i}=K \sum_{j=1}^{N} q_{i j}\left(x_{j}-x_{i}\right)
$$

where $K \in \mathbb{R}^{m \times n}$ is a constant control gain matrix to be designed later. From the relationship between $Q$ and $L$, it is easy to see that

$$
u_{i}=-K \sum_{j=1}^{N} l_{i j} x_{j} .
$$

The closed-loop system is then described by

$$
\dot{x}=\left(I_{N} \otimes A-L \otimes B K\right) x+\Phi(x)
$$

where

$$
x=\left[\begin{array}{c}
x_{1} \\
x_{2} \\
\vdots \\
x_{N}
\end{array}\right], \Phi(x)=\left[\begin{array}{c}
\phi\left(x_{1}\right) \\
\phi\left(x_{2}\right) \\
\vdots \\
\phi\left(x_{N}\right)
\end{array}\right]
$$

and $\otimes$ denotes the Kronicker product of matrices.

Remark 2 : The control input designed in (2) for $i$ th-subsystem uses the relative state information from the subsystems that are connected to this subsystem. In this sense, the control design for each subsystem only uses the relative information of the subsystems in the neighbourhood of this subsystem, and it does not require the information of the entire system.

Let us define $r \in \mathbb{R}^{N}$ as the left eigenvector of $L$ corresponding to the eigenvalue at 0 , that is, $r^{T} L=0$, and furthermore, we set $r^{T} \mathbf{1}=1$.

In the following lemma, we show the state transformation to reveal block diagonal structure of the transformed Laplacian matrix. 
Lemma 3.1: For a Laplacian matrix that satisfies Assumption 1, there exits a similarity transformation $T$ with the first column of $T, T_{1}=\mathbf{1}$ such that

$$
T^{-1} L T=J
$$

with $J$ being a block diagonal matrix in the real Jordan form

$$
J=\left[\begin{array}{lllllll}
0 & & & & & & \\
& J_{1} & & & & & \\
& & \ddots & & & & \\
& & & J_{p} & & & \\
& & & J_{p+1} & & \\
& & & & & \ddots & \\
& & & & & & J_{q}
\end{array}\right]
$$

where $J_{k} \in \mathbb{R}^{n_{k}}$ for $k=1, \ldots, p$ are the Jordan blocks for real eigenvalues $\lambda_{k}>0$ with the multiplicity $n_{k}$ in the form

$$
J_{k}=\left[\begin{array}{cccccc}
\lambda_{k} & 1 & & & \\
& \lambda_{k} & 1 & & \\
& & \ddots & \ddots & \\
& & & \lambda_{k} & 1 \\
& & & & \lambda_{k}
\end{array}\right]
$$

and $J_{k} \in \mathbb{R}^{2 n_{k}}$ for $k=p+1, \ldots, q$ are the Jordan blocks for conjugate eigenvalues $\alpha_{k} \pm j \beta_{k}$, $\alpha_{k}>0$ and $\beta_{k}>0$, with the multiplicity $n_{k}$ in the form

$$
J_{k}=\left[\begin{array}{ccccc}
\mu\left(\alpha_{k}, \beta_{k}\right) & I_{2} & & & \\
& \mu\left(\alpha_{k}, \beta_{k}\right) & I_{2} & & \\
& & \ddots & \ddots & \\
& & & \mu\left(\alpha_{k}, \beta_{k}\right) & I_{2} \\
& & & & \mu\left(\alpha_{k}, \beta_{k}\right)
\end{array}\right]
$$

with $I_{2}$ the identity matrix in $\mathbb{R}^{2 \times 2}$ and

$$
\mu\left(\alpha_{k}, \beta_{k}\right)=\left[\begin{array}{cc}
\alpha_{k} & \beta_{k} \\
-\beta_{k} & \alpha_{k}
\end{array}\right] \in \mathbb{R}^{2 \times 2}
$$

Proof From Theorem 3.4.5 of Horn and Johnson (1990), the real Jordan form exists for $L$ with the similarity transformation matrix $T$ invertible. It can be shown from Assumption 1, we know that 0 is a simple eigenvalue, and furthermore from the Gershgorin theorem (Theorem 6.1.1, Horn and Johnson (1990)), we know that all the other eigenvalues are with positive real parts. We can re-arrange $T$ such that 0 will be the first element in $J$. Furthermore, we can choose $T$ with the first column of $T, T_{1}=\mathbf{1}$ and therefore first row of $T^{-1}, T_{(1)}^{-1}=r^{T}$.

Based on the vector $r$, we can introduce a state transformation

$$
\xi_{i}=x_{i}-\sum_{j=1}^{N} r_{j} x_{j}
$$


for $i=1, \ldots, N$. With

$$
\xi=\left[\xi_{1}^{T}, \ldots \xi_{N}^{T}\right]
$$

the state transformation is given by

$$
\begin{aligned}
\xi & =x-\left(\left(\mathbf{1} r^{T}\right) \otimes I_{n}\right) x \\
& =M \otimes I_{n} x
\end{aligned}
$$

where

$$
M=I_{N}-\mathbf{1} r^{T}
$$

It can be shown that the rank of $M$ is $N-1$, and it has $N-1$ eigenvalues at 1 and one eigenvalue at 0 . Furthermore, it can be seen that the eigenvector corresponding to 0 is $\mathbf{1}$, by $M \mathbf{1}=0$. Therefore, we can conclude that the consensus control is achieved when $\xi=0$, as $\xi=0$ implies that $x_{1}=x_{2}=\ldots=x_{N}$.

The dynamics of $\xi$ can then be obtained as

$$
\dot{\xi}=\left(I_{N} \otimes A-L \otimes B K\right) \xi+\left(M \otimes I_{n}\right) \Phi(x) .
$$

To exploit the structure of $L$, let us introduce another state transformation

$$
\eta=T^{-1} \otimes I_{n} \xi
$$

Its dynamics are obtained as

$$
\dot{\eta}=\left(I_{N} \otimes A-J \otimes B K\right) \eta+\Psi(x)
$$

where

$$
\Psi(x)=\left(\left(T^{-1} M\right) \otimes I_{n}\right) \Phi(x) .
$$

Hence if we can design a control gain matrix $K$ to ensure that $\eta$ converges to zero asymptotically, the consensus control is achieved. For the notational convenience, let us partition the state variable $\eta$ and the nonlinear term $\Psi$ as

$$
\eta=\left[\begin{array}{c}
\eta_{1} \\
\eta_{2} \\
\vdots \\
\eta_{N}
\end{array}\right], \Psi(x)=\left[\begin{array}{c}
\psi_{1}(x) \\
\psi_{2}(x) \\
\vdots \\
\psi_{N}(x)
\end{array}\right]
$$

with $\eta_{i} \in \mathbb{R}^{n}$ and $\psi_{i}: \mathbb{R}^{n \times N} \rightarrow \mathbb{R}^{n}$ for $i=1, \ldots, N$. Then from (11) and (9), we have

$$
\begin{aligned}
\eta_{1} & =r^{T} \otimes I_{n} \xi \\
& =\left(r^{T} M\right) \otimes I_{n} x \\
& \equiv 0 .
\end{aligned}
$$

The nonlinear term $\Psi(x)$ in the transformed system dynamic model (12) is expressed as a function of the state $x$. For the stability analysis, we need to establish a bound of the nonlinear function in terms of the transformed state $\eta$. The following lemma gives a bound of $\psi_{i}$. 
Lemma 3.2: $\quad$ For nonlinear element $\psi_{i}$ of the nonlinear term $\Psi$ in the transformed system dynamic model (12), a bound can be established in terms of the state $\eta$ as shown by

$$
\left\|\psi_{i}\right\| \leq \frac{\gamma_{0}}{\sqrt{N-1}}\|\eta\|
$$

with

$$
\gamma_{0}=2 \sqrt{N(N-1)} \gamma\|r\| \lambda_{\sigma}\left(T^{-1}\right)\|T\|_{F}
$$

where $\lambda_{\sigma}\left(T^{-1}\right)$ denotes the singular value of $T^{-1}$ and $\|T\|_{F}$ denotes the Frobenius norm of $T$.

Proof Based on the state transformations (9) and (11), we have

$$
\psi_{i}(x)=\left(\tau_{i} \otimes I_{n}\right) \mu
$$

where $\tau_{i}$ denotes the $i$ th row of $T^{-1}$ and

$$
\mu=\left(M \otimes I_{n}\right) \Phi
$$

Hence we have

$$
\left\|\psi_{i}(x)\right\| \leq \lambda_{\sigma}\left(T^{-1}\right)\|\mu\|
$$

If we partition $\mu$ in the same way as $\xi$ and $\eta$, we can write, for $j=1, \ldots, N$,

$$
\begin{aligned}
\mu_{j} & =\phi\left(x_{j}\right)-\sum_{k=1}^{N} r_{k} \phi\left(x_{k}\right) \\
& =\sum_{k=1}^{N} r_{k}\left(\phi\left(x_{j}\right)-\phi\left(x_{k}\right)\right) .
\end{aligned}
$$

It then follows that

$$
\begin{aligned}
\left\|\mu_{j}\right\| & \leq \sum_{k=1}^{N}\left|r_{k}\right|\left\|\phi\left(x_{j}\right)-\phi\left(x_{k}\right)\right\| \\
& \leq \gamma \sum_{k=1}^{N}\left|r_{k}\right|\left\|x_{j}-x_{k}\right\| .
\end{aligned}
$$

Again from the state transformations (9) and (11), we have

$$
\begin{aligned}
x_{j}-x_{k} & =\xi_{j}-\xi_{k} \\
& =\left(t_{j}-t_{k}\right) \otimes I_{n} \eta
\end{aligned}
$$


where $t_{j}$ denotes the $j$ th row of $T$. From (14) and (15), we can obtain that

$$
\begin{aligned}
\left\|\mu_{j}\right\| & \leq \gamma \sum_{k=1}^{N}\left|r_{k}\right|\left(\left\|t_{j}\right\|+\left\|t_{k}\right\|\right)\|\eta\| \\
& \leq \gamma\|\eta\|\left(\sum_{j=1}^{N}\left|r_{k}\right|\left\|t_{j}\right\|+\sum_{j=1}^{N}\left|r_{k}\right|\left\|t_{k}\right\|\right) \\
& \leq \gamma\|\eta\|\left(\sum_{j=1}^{N}\left|r_{k}\right|\left\|t_{j}\right\|+\|r\|\|T\|_{F}\right) .
\end{aligned}
$$

From (16), we have

$$
\begin{aligned}
\|\mu\|^{2} & =\sum_{j=1}^{N}\left\|\mu_{j}\right\|^{2} \\
& \left.\leq 2 \gamma^{2}\|\eta\|^{2} \sum_{j=1}^{N}\left(\left\|t_{j}\right\|^{2}\left(\sum_{k=1}^{N}\left|r_{k}\right|\right)^{2}+\|r\|^{2}\|T\|_{F}^{2}\right)\right) \\
& \left.\leq 2 \gamma^{2}\|\eta\|^{2} \sum_{j=1}^{N}\left(\left\|t_{j}\right\|^{2} N\|r\|^{2}+\|r\|^{2}\|T\|_{F}^{2}\right)\right) \\
& =4 N \gamma^{2}\|r\|^{2}\|T\|_{F}^{2}\|\eta\|^{2} .
\end{aligned}
$$

Therefore, we have

$$
\begin{aligned}
\left\|\psi_{i}(x)\right\| & \leq 2 \sqrt{N}\|r\| \lambda_{\sigma}\left(T^{-1}\right)\|T\|_{F}\|\eta\| \\
& =\frac{\gamma_{0}}{\sqrt{N-1}}\|\eta\| .
\end{aligned}
$$

With the control law shown in (2), we design the control gain matrix $K$ as

$$
K=B^{T} P
$$

where $P$ is a positive definite matrix to satisfy certain conditions. In the rest part of the paper, we will use Lyapunov function based analysis to identify a condition for $P$ to ensure that the consensus control objective is achieved by using the control input (2) with the control gain $K$.

The stability analysis will be carried out for $\eta$. Based on the structure of the Laplacian matrix shown in (6), we first show the results for analysis associated with Jordan blocks with real eigenvalues. Denote

$$
N_{k}=1+\sum_{j=1}^{q} n_{j}
$$

for $k=1, \ldots, q$. Note that $N_{q}=N$.

The subsystem state variables $\eta_{i}$ for $i=2$ to $i=N_{p}$ are the state variables which are associated with the Jordan blocks of real eigenvalues, and $\eta_{i}$ for $i=N_{p}+1$ to $i=N$ are with Jordan blocks of complex eigenvalues. 
Within each real Jordan block $J_{k}$, for $k \leq p$, we have the dynamics given by

$$
\dot{\eta}_{i}=\left(A-\lambda_{k} B B^{T} P\right) \eta_{i}-B B^{T} P \eta_{i+1}+\psi_{i}(x)
$$

for $i=N_{k-1}+1, \ldots, N_{k}-1$, and

$$
\dot{\eta}_{i}=\left(A-\lambda_{k} B B^{T} P\right) \eta_{i}+\psi_{i}(x)
$$

for $i=N_{k}$.

Let $W_{i}=\eta_{i}^{T} P \eta_{i}$. For $i=N_{k-1}+1, \ldots, N_{k}-1$, we obtain that

$$
\begin{aligned}
\dot{W}_{i}= & \eta_{i}^{T}\left(A^{T} P+P A-2 \lambda_{k} P B B^{T} P\right) \eta_{i} \\
& -2 \eta_{i}^{T} P B B^{T} P \eta_{i+1}+2 \eta_{i}^{T} P \psi_{i} \\
\leq & \eta_{i}^{T}\left(A^{T} P+P A-\left(2 \lambda_{k}-\frac{1}{\sigma}\right) P B B^{T} P+\kappa P P\right) \eta_{i} \\
& +\sigma \eta_{i+1}^{T} P B B^{T} P \eta_{i+1}+\frac{1}{\kappa}\left\|\psi_{i}\right\|^{2}
\end{aligned}
$$

where $\sigma$ and $\kappa$ are two positive real numbers and we have used the inequality

$$
2 a^{T} b \leq \kappa a^{T} a+\frac{1}{\kappa} b^{T} b
$$

for vectors $a$ and $b$ with the same dimension.

Considering the derivative of $W_{i}+\sigma^{2} W_{i+1}$ for $i=N_{k-1}+1, \ldots, N_{k}-2$, we can obtain that

$$
\begin{aligned}
\dot{W}_{i}+\sigma^{2} \dot{W}_{i+1} \leq & \eta_{i}^{T}\left(A^{T} P+P A-\left(2 \lambda_{k}-\frac{1}{\sigma}\right) P B B^{T} P+\kappa P P\right) \eta_{i}+\frac{1}{\kappa}\left\|\psi_{i}\right\|^{2} \\
& +\sigma^{2}\left(\eta_{i+1}^{T}\left(A^{T} P+P A-2\left(\lambda_{k}-\frac{1}{\sigma}\right) P B B^{T} P+\kappa P P\right) \eta_{i+1}+\frac{1}{\kappa}\left\|\psi_{i+1}\right\|^{2}\right) \\
& +\sigma^{3} \eta_{i+2}^{T} P B B^{T} P \eta_{i+2} .
\end{aligned}
$$

Similarly for $i=N_{k}$, we have

$$
\dot{W}_{i} \leq \eta_{i}^{T}\left(A^{T} P+P A-2 \lambda_{k} P B B^{T} P+\kappa P P\right) \eta_{i}+\frac{1}{\kappa}\left\|\psi_{i}\right\|^{2} .
$$

Let

$$
V_{k}=\sum_{j=1}^{n_{k}} \sigma^{2(j-1)} W_{j+N_{k-1}} .
$$

From (21) and (22), we obtain that

$$
\begin{aligned}
\dot{V}_{k} \leq & \sum_{j=1}^{n_{k}} \sigma^{2(j-1)}\left(\eta_{j+N_{k-1}}^{T}\left(A^{T} P+P A-2\left(\lambda_{k}-\frac{1}{\sigma}\right) P B B^{T} P+\kappa P P\right) \eta_{j+N_{k-1}}+\frac{1}{\kappa}\left\|\psi_{j+N_{k-1}}\right\|^{2}\right) \\
& -\frac{1}{\sigma} \eta_{1+N_{k-1}}^{T} P B B^{T} P \eta_{1+N_{k-1}}-\sigma^{2 n_{k}-3} \eta_{N_{k}-1}^{T} P B B^{T} P \eta_{N_{k}-1} .
\end{aligned}
$$

For the state variables associated with the Jordan blocks $J_{k}$ for $k>p$ corresponding to complex eigenvalues, we consider the dynamics of the state variables in pairs. For notational convenience, 
let

$$
\begin{aligned}
& i_{1}(j)=N_{k-1}+2 j-1, \\
& i_{2}(j)=N_{k-1}+2 j,
\end{aligned}
$$

for $j=1, \ldots, n_{k} / 2$. The dynamics of $\eta_{i_{1}}$ and $\eta_{i_{2}}$ for $j=1, \ldots, n_{k} / 2-1$ are expressed by

$$
\begin{aligned}
& \left.\dot{\eta}_{i_{1}}=\left(A-\alpha_{k} B B^{T} P\right) \eta_{i_{1}}-\beta_{k} B B^{T} P\right) \eta_{i_{2}}-B B^{T} P \eta_{i_{1}+2}+\psi_{i_{1}} \\
& \left.\dot{\eta}_{i_{2}}=\left(A-\alpha_{k} B B^{T} P\right) \eta_{i_{2}}+\beta_{k} B B^{T} P\right) \eta_{i_{1}}-B B^{T} P \eta_{i_{2}+2}+\psi_{i_{2}} .
\end{aligned}
$$

For $j=n_{k} / 2$, we have

$$
\begin{aligned}
& \left.\dot{\eta}_{i_{1}}=\left(A-\alpha_{k} B B^{T} P\right) \eta_{i_{1}}-\beta_{k} B B^{T} P\right) \eta_{i_{2}}+\psi_{i_{1}} \\
& \left.\dot{\eta}_{i_{2}}=\left(A-\alpha_{k} B B^{T} P\right) \eta_{i_{2}}+\beta_{k} B B^{T} P\right) \eta_{i_{1}}+\psi_{i_{2}} .
\end{aligned}
$$

For the derivative of $W_{i_{1}}+W_{i_{2}}$ for $j=n_{k} / 2$, we can obtain, by noticing the cancellation of the terms associated with $\beta_{k}$, that

$$
\begin{aligned}
\dot{W}_{i_{1}}+\dot{W}_{i_{2}} \leq & \eta_{i_{1}}^{T}\left(A^{T} P+P A-2 \alpha_{k} P B B^{T} P+\kappa P P\right) \eta_{i_{1}} \\
& +\eta_{i_{2}}^{T}\left(A^{T} P+P A-2 \alpha_{k} P B B^{T} P+\kappa P P\right) \eta_{i_{2}} \\
& +\frac{1}{\kappa}\left(\left\|\psi_{i_{1}}\right\|^{2}+\left\|\psi_{i_{2}}\right\|^{2}\right) .
\end{aligned}
$$

For $j=1, \ldots, n_{k} / 2-1$, we can obtain that

$$
\begin{aligned}
\dot{W}_{i_{1}}+\dot{W}_{i_{2}} \leq & \eta_{i_{1}}^{T}\left(A^{T} P+P A-\left(2 \alpha_{k}-\frac{1}{\sigma}\right) P B B^{T} P+\kappa P P\right) \eta_{i_{1}} \\
& +\eta_{i_{2}}^{T}\left(A^{T} P+P A-\left(2 \alpha_{k}-\frac{1}{\sigma}\right) P B B^{T} P+\kappa P P\right) \eta_{i_{2}} \\
& +\sigma \eta_{i_{1}+2}^{T} P B B^{T} P \eta_{i_{1}+2}+\sigma \eta_{i_{2}+2}^{T} P B B^{T} P \eta_{i_{2}+2}+\frac{1}{\kappa}\left(\left\|\psi_{i_{1}}\right\|^{2}+\left\|\psi_{i_{2}}\right\|^{2}\right) .
\end{aligned}
$$

Let

$$
V_{k}=\sum_{j=1}^{n_{k} / 2} \sigma^{2(j-1)}\left(W_{i_{1}(j)}+W_{i_{2}(j)}\right)
$$


for $k=p+1, \ldots, q$. Similar to the case for the blocks with real eigenvalues, we can obtain that

$$
\begin{aligned}
\dot{V}_{k} \leq & \sum_{j=1}^{n_{k} / 2} \sigma^{2(j-1)}\left(\eta_{2 j-1+N_{k-1}}^{T}\left(A^{T} P+P A-2\left(\alpha_{k}-\frac{1}{\sigma}\right) P B B^{T} P+\kappa P P\right) \eta_{2 j-1+N_{k-1}}\right. \\
& +\eta_{2 j+N_{k-1}}^{T}\left(A^{T} P+P A-2\left(\alpha_{k}-\frac{1}{\sigma}\right) P B B^{T} P+\kappa P P\right) \eta_{2 j+N_{k-1}} \\
& \left.+\frac{1}{\kappa}\left(\left\|\psi_{2 j-1+N_{k-1}}\right\|^{2}+\left\|\psi_{2 j+N_{k-1}}\right\|^{2}\right)\right) \\
& -\frac{1}{\sigma}\left(\eta_{1+N_{k-1}}^{T} P B B^{T} P \eta_{1+N_{k-1}}+\eta_{2+N_{k-1}}^{T} P B B^{T} P \eta_{2+N_{k-1}}\right) \\
& -\sigma^{n_{k}-3}\left(\eta_{N_{k}-1}^{T} P B B^{T} P \eta_{N_{k}-1}+\eta_{N_{k}}^{T} P B B^{T} P \eta_{N_{k}}\right) .
\end{aligned}
$$

Finally, let

$$
V=\sum_{k=1}^{q} V_{k} .
$$

From (23) and (27), we can obtain that

$$
\begin{aligned}
\dot{V} \leq & \sum_{k=1}^{p} \sum_{j=1}^{n_{k}} \sigma^{2(j-1)}\left(\eta_{j+N_{k-1}}^{T}\left(A^{T} P+P A-2\left(\lambda_{k}-\frac{1}{\sigma}\right) P B B^{T} P+\kappa P P\right) \eta_{j+N_{k-1}}+\frac{1}{\kappa}\left\|\psi_{j+N_{k-1}}\right\|^{2}\right) \\
& +\sum_{k=q+1}^{p} \sum_{j=1}^{n_{k} / 2} \sigma^{2(j-1)}\left(\eta_{2 j-1+N_{k-1}}^{T}\left(A^{T} P+P A-2\left(\alpha_{k}-\frac{1}{\sigma}\right) P B B^{T} P+\kappa P P\right) \eta_{2 j-1+N_{k-1}}\right. \\
& +\eta_{2 j+N_{k-1}}^{T}\left(A^{T} P+P A-2\left(\alpha_{k}-\frac{1}{\sigma}\right) P B B^{T} P+\kappa P P\right) \eta_{2 j+N_{k-1}} \\
& \left.+\frac{1}{\kappa}\left(\left\|\psi_{2 j-1+N_{k-1}}\right\|^{2}+\left\|\psi_{2 j+N_{k-1}}\right\|^{2}\right)\right) \\
& -\sum_{k=1}^{p}\left(\frac{1}{\sigma} \eta_{1+N_{k-1}}^{T} P B B^{T} P \eta_{1+N_{k-1}}+\sigma^{2 n_{k}-3} \eta_{N_{k}}^{T} P B B^{T} P \eta_{N_{k}}\right) \\
& -\sum_{k=q+1}^{p}\left(\frac{1}{\sigma} \eta_{1+N_{k-1}}^{T} P B B^{T} P \eta_{1+N_{k-1}}+\frac{1}{\sigma} \eta_{2+N_{k-1}}^{T} P B B^{T} P \eta_{2+N_{k-1}}\right. \\
& \left.+\sigma^{n_{k}-3} \eta_{N_{k}-1}^{T} P B B^{T} P \eta_{N_{k}-1}+\sigma^{n_{k}-3} \eta_{N_{k}}^{T} P B B^{T} P \eta_{N_{k}}\right) .
\end{aligned}
$$

The above expression can be used for stability analysis of consensus control of nonlinear systems with Lipschitz nonlinear systems. The following theorem summarises the results.

Theorem 3.3: For a network connected dynamic system (1) with the associated Laplacian matrix that satisfies Assumption 1, the consensus control problem can be solved by the control design (2) with the control gain $K=B^{T} P$ with the positive definite matrix $P$ specified in one of the following two cases:

1. If the eigenvalues of the Laplacian matrix distinct, i.e., $n_{k}=1$ for $k=1, \ldots, q$, the matrix $P$ 
satisfies

$$
A^{T} P+P A-2 \alpha P B B^{T} P+\kappa P P+\frac{\gamma_{0}^{2}}{\kappa} I_{n}<0
$$

with $\kappa$ being any positive real number, and $\alpha=\min \left\{\lambda_{1}, \ldots, \lambda_{p}, \alpha_{p+1}, \ldots, \alpha_{q}\right\}$.

2. If the Laplacian matrix have multiple eigenvalues, i.e., $n_{k}>1$ for any $k \in\{1, \ldots, q\}$, the matrix P satisfies

$$
A^{T} P+P A-2(\alpha-1) P B B^{T} P+\kappa P P+\frac{\gamma_{0}^{2}}{\kappa} I_{n}<0
$$

with $\kappa$ being any positive real number.

Proof The case 1 . When the eigenvalues are distinct, from (29), we can obtain that

$$
\dot{V} \leq \sum_{i=2}^{N}\left(\eta_{i}^{T}\left(A^{T} P+P A-2 \alpha P B B^{T} P+\kappa P P\right) \eta_{i}+\frac{1}{\kappa}\left\|\psi_{i}\right\|^{2}\right) .
$$

From Lemma 3.2, we have

$$
\begin{aligned}
\dot{V} & \leq \sum_{i=2}^{N}\left(\eta_{i}^{T}\left(A^{T} P+P A-2 \alpha P B B^{T} P+\kappa P P\right) \eta_{i}+\frac{1}{\kappa} \frac{\gamma_{0}^{2}}{N-1}\|\eta\|^{2}\right) \\
& \leq \sum_{i=2}^{N}\left(\eta_{i}^{T}\left(A^{T} P+P A-2 \alpha P B B^{T} P+\kappa P P+\frac{\gamma_{0}^{2}}{\kappa} I_{n}\right) \eta_{i}\right)
\end{aligned}
$$

where we have used $\|\eta\|^{2}=\sum_{i=2}^{N}\left\|\eta_{i}\right\|^{2}$. From (32) and (30), we can show that $\dot{V}<0$. Hence we conclude that $\eta$ converges to zero asymptotically.

The case 2. Setting $\sigma=1$, and from (29), we can obtain that

$$
\dot{V} \leq \sum_{i=2}^{N}\left(\eta_{i}^{T}\left(A^{T} P+P A-2(\alpha-1) P B B^{T} P+\kappa P P\right) \eta_{i}+\frac{1}{\kappa}\left\|\psi_{i}\right\|^{2}\right) .
$$

Using the result of Lemma 3.2, and following a similar procedure as in the proof for the case 1, we can show that $\dot{V}<0$. Hence we conclude that $\eta$ converges to zero asymptotically.

The stability analysis for Lipschitz nonlinear systems can clearly be applied to linear systems. Although the result for consensus control of linear systems is known in literature, it would still be of interest to establish the result using the method based on the real Jordan form that is developed in this paper for Lipschitz nonlinear systems.

Corollary 3.4: For a network connected dynamic system (1) with the associated Laplacian matrix that satisfies Assumption 1, if the system does not have nonlinear terms (corresponding to $\gamma=0)$, the consensus control problem can be solved by the control design (2) with the control gain $K=B^{T} P$ with the positive definite matrix $P$ satisfying

$$
A^{T} P+P A-2 \alpha P B B^{T} P<0
$$

where $\alpha=\min \left\{\lambda_{1}, \ldots, \lambda_{p}, \alpha_{p+1}, \ldots, \alpha_{q}\right\}$. 
Proof When there is no nonlinear term, by ignoring the terms associated with $\kappa$ in (29), we can obtain that

$$
\begin{aligned}
\dot{V} \leq & \sum_{k=1}^{p} \sum_{j=1}^{n_{k}} \sigma^{2(j-1)}\left(\eta_{j+N_{k-1}}^{T}\left(A^{T} P+P A-2\left(\lambda_{k}-\frac{1}{\sigma}\right) P B B^{T} P\right) \eta_{j+N_{k-1}}\right) \\
& +\sum_{k=q+1}^{p} \sum_{j=1}^{n_{k} / 2} \sigma^{2(j-1)}\left(\eta _ { 2 j - 1 + N _ { k - 1 } } ^ { T } \left(A^{T} P+P A-2\left(\alpha_{k}-\frac{1}{\sigma}\right) P B B^{T} P \eta_{2 j-1+N_{k-1}}\right.\right. \\
& +\eta_{2 j+N_{k-1}}^{T}\left(A^{T} P+P A-2\left(\alpha_{k}-\frac{1}{\sigma}\right) P B B^{T} P \eta_{2 j+N_{k-1}}\right)
\end{aligned}
$$

From (33), there exist a small positive real number $\epsilon$ such that

$$
A^{T} P+P A-2 \alpha P B B^{T} P+\epsilon I<0 .
$$

Setting $\sigma>2 \lambda_{\sigma}\left(P B B^{T} P\right) / \epsilon$, from (34) and (35), we can show that $\dot{V}<0$. Hence we conclude that $\eta$ converges to zero asymptotically.

Remark 3 : The result shown in Corollary 3.4 has been established in literature using a different method ( $\mathrm{Li}$ et al. 2010). Here we use a Lyapunov function in real domain to prove the result for linear systems, which is the the same framework used for the nonlinear systems with Lipschitz nonlinearity. It is clear in the case 2 of Theorem 3.3 that the choice of the value of $\sigma$ will affect the condition specified for achieving the global consensus control. Based on the current framework for analysis, $\sigma=1$ would be an optimal choice. Also note that eigenstructure of the Laplacian matrix is needed for the control design, as we need to see which case to apply in Theorem 3.3. The methodology of using a Lyapunov function in real domain may also be instrumental in stability analysis of other consensus control systems.

Remark 4: The conditions shown in (33), (30) and (31) can be formulated as linear matrix inequalities (LMIs). The condition is (33) is equivalent to

$$
P^{-1} A^{T}+A P^{-1}-2 \alpha B B^{T}<0
$$

and a solution of $P^{-1}$ is guaranteed if $(A, B)$ is controllable. The condition (30) is equivalent to

$$
P^{-1} A^{T}+A P^{-1}-2 \alpha B B^{T}+\kappa I_{n}+\frac{\gamma_{0}^{2}}{\kappa} P^{-1} P^{-1}<0
$$

which is further equivalent to the LMI

$$
\left[\begin{array}{cc}
P^{-1} A^{T}+A P^{-1}-2 \alpha B B^{T}+\kappa I_{n} & P^{-1} \\
P^{-1} & -\frac{\kappa}{\gamma_{0}^{2}} I_{n}
\end{array}\right]<0 .
$$

The feasibility and a possible solution of $P^{-1}$ can be easily checked and obtained from standard LMI routines. The condition (31) can be checked in the same way as (30).

Remark 5: Theorem 3.3 shows the conditions that guarantee a solution to the consensus control problem, but does not specify the consensus trajectories. Let us define a state transform

$$
z=T^{-1} \otimes I_{n} x
$$


The dynamics of $z$ can be obtained as

$$
\dot{z}=\left(I_{N} \otimes A-J \otimes B K\right) z+T^{-1} \otimes I_{n} \Phi(x)
$$

We can partition $z$ in the same way as $x$, i.e., with

$$
z=\left[\begin{array}{c}
z_{1} \\
z_{2} \\
\vdots \\
z_{N}
\end{array}\right]
$$

we then have

$$
\dot{z}_{1}=A z_{1}+r^{T} \otimes I_{n} \Phi(x)
$$

Note $z_{1}=r^{T} \otimes I_{n} x$. If there is no nonlinear term, $z_{1}$ specifies the consensus trajectory, as shown by $\mathrm{Li}$ et al. (2010) with $z_{1}(t)=e^{A t} z_{1}(0)$. The explicit expression of the consensus trajectory is not available in general, due to the involvement of the nonlinear terms, but it is clear that the nonlinear function $\phi\left(x_{i}\right)$ have an influence on it. The simulation results clearly show this in the example later.

\section{Simulation Study}

In this section, we will show some details of the proposed consensus control design through an example. The system under consideration is a connection of 4 subsystems, each of them is described by a second order state space model as

$$
\dot{x}_{i}=\left[\begin{array}{cc}
-1 & 1 \\
0 & 0
\end{array}\right] x_{i}+\left[\begin{array}{l}
0 \\
1
\end{array}\right] u+\left[\begin{array}{c}
0.05 \sin \left(C x_{i}\right) \\
0
\end{array}\right] u
$$

where

$$
C=\left[\begin{array}{ll}
1 & 0
\end{array}\right]
$$

The adjacent matrix is given by

$$
Q=\left[\begin{array}{llll}
0 & 0 & 0 & 1 \\
1 & 0 & 0 & 0 \\
0 & 0 & 0 & 1 \\
0 & 1 & 0 & 0
\end{array}\right]
$$

and the resultant Laplacian matrix is obtained as

$$
L=\left[\begin{array}{cccc}
1 & 0 & 0 & -1 \\
-1 & 1 & 0 & 0 \\
0 & 0 & 1 & -1 \\
0 & -1 & 0 & 1
\end{array}\right]
$$


The eigenvalues of $L$ are $\left\{0,1, \frac{3 \pm \sqrt{3} j}{2}\right\}$ and therefore Assumption 1 is satisfied. Furthermore, the eigenvalues are distinct. We can obtain that

$$
J=\left[\begin{array}{cccc}
0 & 0 & 0 & 0 \\
0 & 1 & 0 & 0 \\
0 & 0 & \frac{3}{2} & \frac{\sqrt{3}}{2} \\
0 & 0 & -\frac{\sqrt{3}}{2} & \frac{3}{2}
\end{array}\right]
$$

with the matricies

$$
T=\left[\begin{array}{cccc}
1 & 0 & \frac{1}{2} & \frac{\sqrt{3}}{2} \\
1 & 0 & -1 & 0 \\
1 & -2 & \frac{1}{2} & \frac{\sqrt{3}}{2} \\
1 & 0 & \frac{1}{2} & -\frac{\sqrt{3}}{2}
\end{array}\right]
$$

and

$$
T^{-1}=\left[\begin{array}{cccc}
\frac{1}{3} & \frac{1}{3} & 0 & \frac{1}{3} \\
\frac{1}{2} & 0 & -\frac{1}{2} & 0 \\
\frac{1}{3} & -\frac{2}{3} & 0 & \frac{1}{3} \\
\frac{\sqrt{3}}{3} & 0 & 0 & -\frac{\sqrt{3}}{3}
\end{array}\right]
$$

Thus we have

$$
r^{T}=\left[\begin{array}{llll}
\frac{1}{3} & \frac{1}{3} & 0 & \frac{1}{3}
\end{array}\right]
$$

Remark 6 : For this example, the Laplacian matrix $L$ is asymmetric, as the connection graph is a directed one, which implies that the results shown in (Li et al. 2013) cannot be applied. It is worth noting that the third element of the left eigenvector $r$ is zero. This suggests that the Lyapunov function used in ( $\mathrm{Li}$ et al. 2012) cannot be used for the stability analysis here.

The nonlinear function $0.05 \sin \left(C x_{i}\right)$ in the system dynamics is globally Lipschitz, and we obtain $\gamma=0.05$ and $\gamma_{0}=0.6734$. Based on the Laplacian matrix $L$, we have $\alpha=1$. A positive definite matrix $P$ to satisfy (30) can be obtained, with $\kappa=1.0$, as

$$
P=\left[\begin{array}{cc}
2.0527 & 2.7372 \\
2.7372 & 12.3170
\end{array}\right]
$$

and the control gain is obtained as

$$
K=[2.737212 .3170]
$$

Simulation study has been carried out with the results shown in Figures 1 and 2 for the states 1 and 2 of the subsystems.

It is noted that the condition specified for (30) is a sufficient condition for the control gain to achieve consensus control when there are nonlinearities in the system, and this condition can be very conservative in the control gain design for a given Lipschitz nonlinear function. Indeed, the same control gain can possibly achieve consensus control for nonlinearities with a Lipschitz constant much bigger than 0.05. Simulation results for

$$
\phi\left(x_{i}\right)=\left[\begin{array}{c}
\sin \left(C x_{i}\right) \\
0
\end{array}\right]
$$




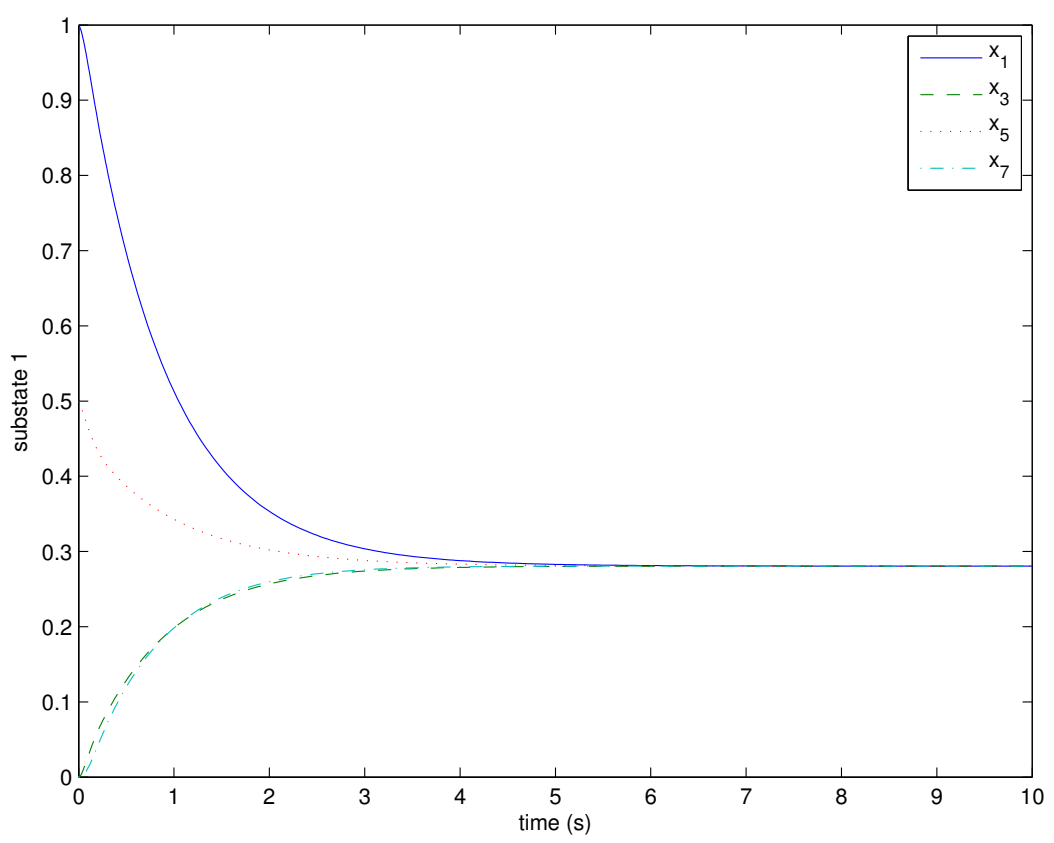

Figure 1. The state 1 of subsystems.

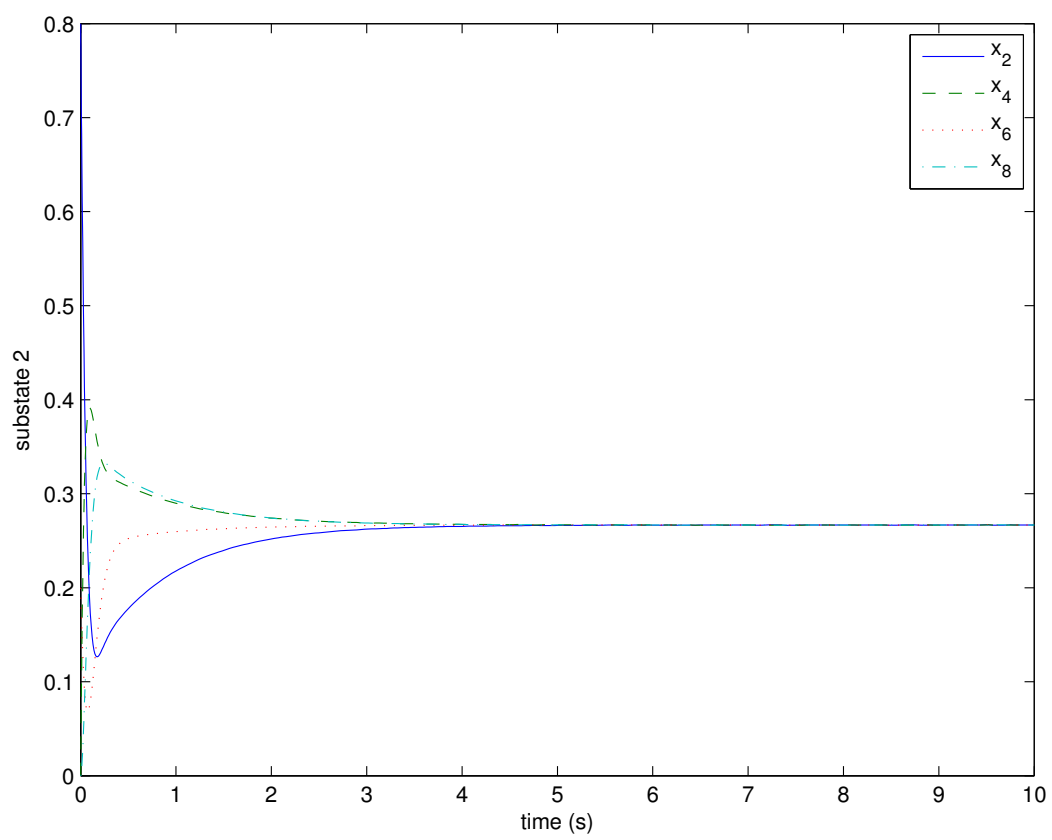

Figure 2. The state 2 of subsystems.

are shown in Figures 3 and 4 . The results suggest that consensus control can still be achieved for a stronger nonlinearity with the same control gain, but it took a bit longer time due to the stronger nonlinearity. It is also noticed that the consensus trajectories are now different from those shown in Figures 1 and 2, duo the difference in the nonlinear term. 


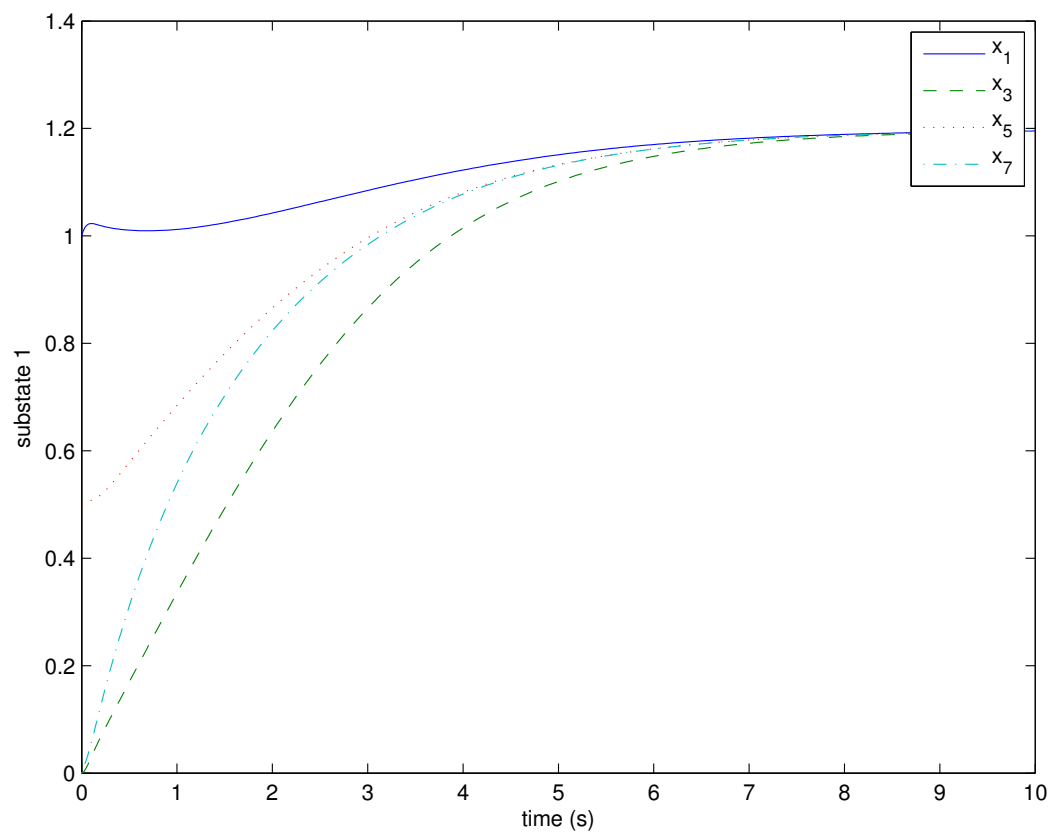

Figure 3. The state 1 of subsystems with stronger nonlinearity.

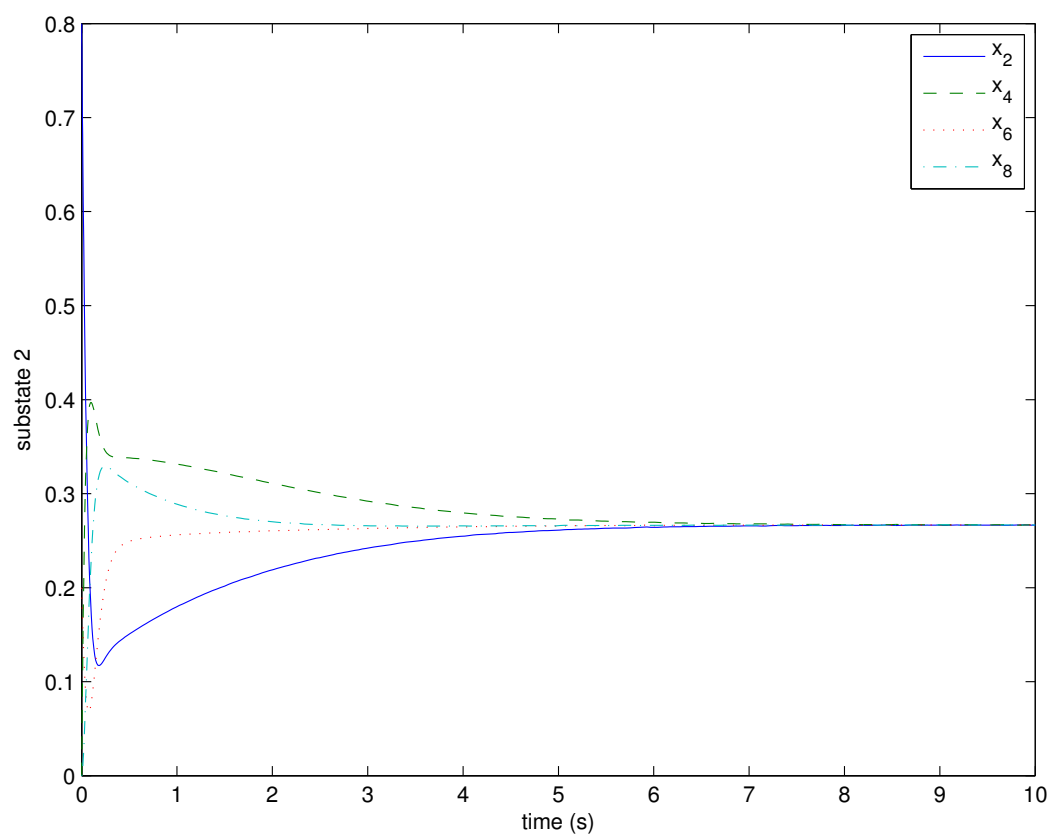

Figure 4 . The state 2 of subsystems with stronger nonlinearity.

\section{Conclusion}

In this paper, we have developed a framework for stability analysis of consensus control systems using Lyapunov functions in real domain. Using this framework, we have investigated the influences of nonlinearities in consensus control. After a careful study of the structure of Laplacian matrix in real Jordan form, conditions based on the Lipschitz constant have been identified for 
a proposed linear control law to tackle Lipschitz nonlinear terms in the system dynamics. It has been shown that a more stringent condition is required when the Laplacian has multiple eigenvalues. The stability analysis shown in this paper is different from the methods shown in (Li et al. 2012, 2013), and can be applied to the systems with more general connections.

In the set up of the presentation, the global stability is analysed for the closed-loop system under the proposed consensus control. This study can be applied to investigate consensus control around operating points with the consideration of stability in a small neighbourhood. In such a case, consensus control can always be achieved provided that the neighbourhood is small enough, because the Lipschitz constants are getting smaller when getting closer to the operating points. In other words, the result presented in this paper can also be used to check the operating region, in which the stability can be ensured by checking the local Lipschitz constants for nonlinear terms that may not even be globally Lipschitz.

\section{References}

Ding, Z. (2012), "Consensus output regulation without state estimation," in The Proceedings of The 10th World Congress on Intelligent Control and Automation, Beijing, China, pp. 23062311.

Ding, Z. (2013), "Consensus output regulation of a class of heterogeneous nonlinear systems," IEEE Trans. Automatic Control, 58, 2648-2653.

Fax, J.A., and Murray, R.M. (2004), "Information flow and cooperative control of vehicle formation," IEEE Trans. Automa. Contr., 49, 1465-1476.

Hong, Y., Hu, J., and Gao, L. (2006), "Tracking control for multi-agent consensus with an active leader and variable topology," Automatica, 42, 1177-1182.

Horn, R.A., and Johnson, C.A., Matrix Analysis, Cambridge, England: Cambridge Univ. Press (1990).

Li, Z., Duan, Z., Chen, G., and Huang, L. (2010), "Consensus of multiagent systems and synchronization of complex networks, a unified viewpoint," IEEE Trans. Circuits Syst. I., 57, 213-224.

Li, Z., Liu, X., Fu, M., and Xie, L. (2012), "Global $H_{\infty}$ consensus of multi-agent systems with Lipschitz non-linear dynamics," IET Control Theory 85 Applications, 6, 2041-2048.

Li, Z., Ren, W., Liu, X., and Fu, M. (2013), "Consensus of multi-agent systems with general linear and Lipschitz nonlinear dynamics using distributed adaptive protocols," IEEE Trans. Automa. Contr., 58, 1786-1791.

Liu, L. (2011), "Robust cooperative output regulation problem for nonlinear multi-agent systems," in Proceedings of 9th IEEE Conference on Control and Automation, Santiago, Chile, pp. 644-649.

Liu, S., Xie, L., and Lewis, F.L. (2011), "Synchronization of multi-agent systems with delayed control input information from neighbor," Automatica, 47, 2152-2164.

Ren, W. (2007), "Multi-vehicle consensus with a time-varying reference state," Systems and Control Letters, 56, 474-483.

R.Olfati-Saber,, and Murray, R.M. (2004), "Consensus problems in networks of agents with switching topology and time-delay," IEEE Trans. Automa. Contr., 49, 1520-1533.

Song, Q., Cao, J., and Yu, W. (2010), "Second-order leader-following consensus of nonlinear multi-agent systems via pinning control," Systems $\&$ Control Letters, 59, 553-562.

$\mathrm{Su}$, Y., and Huang, J. (2012), "Stability of a class of linear switching systems with applications to two consensus problems," IEEE Trans. Automa. Contr., 57, 1420-1430.

Wang, X., Hong, Y., Huang, J., and Jiang, Z.P. (2010), "A distributed control approach to robust output regulation problem for multi-agent linear systems," IEEE Trans. Automa. Contr., 55, $2891-2895$.

Xiang, J., Wei, W., and Li, Y. (2009), "Synchronized output regulation of linear networked systems," IEEE Trans. Automa. Contr., 54, 1336-1341. 
Yu, W., Chen, G., Cao, M., and Kurths, J. (2010), "Second-order consensus for multiagent systems with directed topologies and nonlinear dynamics," IEEE Trans. Systems, Man, and Cybernetics, Part B: Cybernetics,, 40, 881-891. 